\title{
Motivational structure of ayahuasca drinkers in social networks
}

\author{
TOM JOHN WOLFF ${ }^{1}$ and TORSTEN PASSIE ${ }^{2,3}$ \\ ${ }^{1}$ Institut für Public Health und Pflegeforschung, Abteilung 6 Gesundheit und Gesellschaft, University of Bremen, Bremen, Germany \\ ${ }^{2}$ Department of Psychiatry, Social Psychiatry and Psychotherapy, Hannover Medical School, Hannover, Germany \\ ${ }^{3}$ Senckenberg Institute for History and Ethics in Medicine, Goethe-University, Frankfurt am Main, Germany
}

(Received: February 9, 2018; revised manuscript received: June 12, 2018; accepted: September 20, 2018)

\begin{abstract}
Objectives: The international distribution and commodification of ayahuasca has created virtual communicative realms in the Internet in which members intensively exchange personal narratives, advertisements, and beliefs about ayahuasca and its reputed native background. Common consumption standards and beliefs about ayahuasca have developed among western ayahuasca drinkers who are often only loosely connected to the local practice of ayahuasca-shamanism in regions of the upper Amazonas that are not yet influenced by ayahuasca tourism. Those new standards of the ayahuasca tourism are reflected in the Internet. Motivation and expectation of interested ayahuasca tourists gets shaped in Internet forums. Previous studies about the motivation of western ayahuasca drinkers have mainly used heuristic qualitative methods. This study characterizes ayahuasca Internet activists and examines statistically their motivational structure to drink ayahuasca. Methods: Using online data collection and quantitative analysis methods, 40 participants were studied who were active in closed Facebook groups about ayahuasca in the year 2015. Results: The average active members of ayahuasca Facebook groups in this sample seem to be 28-50 years old $( \pm 1 S D)$, higher education degree holders, preexperienced with other psychedelic drugs and other psychospiritual methods, preexperienced with ayahuasca and motivated to drink again, and tend toward the intake within organized events such as shamanic healing ceremonies and retreat seminars. The results suggest that the motivation of Internet activists for ayahuasca consists in majority of four main elements: self-exploration, spiritual purposes, physical health issues, and sensation seeking. Self-exploration and spiritual purposes appear to be the main reasons for drinking ayahuasca. Physical health issues and sensation seeking are minor reasons among this subgroup of western ayahuasca drinkers but may play a larger role in some participants. Conclusions: The motivation of members of social ayahuasca networks in the Internet seems not to be monodimensional but a composite of different aspects of which a subconglomerate of psychospiritual reasons and individual self-development seems to be the most dominant. The findings support motivational differences to local ayahuasca shaman clients in the upper Amazonas region that have been previously described in the ethnologic literature.
\end{abstract}

Keywords: ayahuasca, motivation, social networks, Facebook, motivational structure

\section{INTRODUCTION}

Ayahuasca is a Quechua name of a psychoactive beverage that is mostly made of several species of the vine Banisteriopsis (B. caapi and B. muricata). It usually contains leaves of other plants such as Psychotria viridis, Diplopterys cabrerana, or others (Ott, 1994, 1996). Its use has been reported from more than 70 different indigenous groups (Luna, 2005) as well as from mestizo population of the upper Amazonas (Beyer, 2009; Incayawar, 2007). $\mathrm{N}, \mathrm{N}$-dimethyltryptamine (DMT) has been identified as the main compound. It is prevented from being deaminated by MAO-A-inhibiting beta-carbolines (McKenna, Towers, \& Abbott, 1984; Schenberg et al., 2015). The beverage is orally ingested within a ritualized context for physical healing, divination, social reasons like communicating with distant people or spirits, witchcraft, or identifying sorcery (de Rios, 1972; Luna, 2004) and known to be drunk either exclusively by the medicine man or collectively (Miller-Weisberger, 2000; Schultes, 1983). The current legal situation remains complicated since the drinking practices in various countries exist in legal vacuums or at times contradictory legal conventions (Labate, Cavnar, \& Gearin, 2017). Since the beginning of the 20th century, syncretic ayahuasca religions have developed in Brazil, spread into urban regions since the 1980s, and lately established branches in Japan, Netherlands, USA, and other countries (Barbosa, Mizumoto, Bogenschutz, \& Strassmann, 2012; Santos, Landeira-Fernandez, Strassman, Motta, \& Cruz, 2007). Ayahuasca-shamanism has also been

* Corresponding author: Tom John Wolff, Dipl.-Psych.; Institut für Public Health und Pflegeforschung, Abteilung 6 Gesundheit und Gesellschaft, Prof. Schmidt-Semisch, University of Bremen, Grazer Straße 2, R. 0090, 28359 Bremen, Germany; Phone: +51968867 821; Fax: +49 4212189868 880; E-mail: tiofijo@gmx.de

This is an open-access article distributed under the terms of the Creative Commons Attribution-NonCommercial 4.0 International License, which permits unrestricted use, distribution, and reproduction in any medium for non-commercial purposes, provided the original author and source are credited, a link to the CC License is provided, and changes - if any - are indicated. 
a profession among Christian-mestizo culture long before the western interest arose (Fotiou, 2010). For the first time in 1963, The "Yage Letters" of Burroughs and Ginsberg (1963) brought the beverage to the attention of a small non-scientific international audience. Besides the folkhealing tradition of the upper Amazonas, a flourishing cross-cultural ayahuasca tourism has lately developed during the past 20 years, mainly from the developed North American and other western countries (Fotiou, 2014; Homan, 2017). Touristic activities mainly concentrate around the bigger jungle cities of Peru but also Ecuador and southern Colombia as well as parts of western Brazil. Jungle lodges, often run by foreigners who focus on western ayahuasca drinkers, provide ayahuasca ceremonies in semi-folkloric or traditionalized settings, sometimes along with other touristic and therapeutic activities and longer seminars (Holman, 2011; Znamenski, 2007). Holman (2010) sees evidence for a major transformative and spiritual motivation of western ayahuasca drinkers, besides the adventure and sensation-seeking motivation, the rebellious ideology against western lifestyle, and the romantic projective mechanism of ethno-tourism.

Some authors expressed a rather monocausal perspective on the motivation of ayahuasca tourists. de Rios (1994) and Grunwell (1998) view the search for extraordinary experience as main motivation, which cannot be provided by their own culture. Fotiou (2010) identified the search for the antithesis of western civilization as the common theme behind all kinds of motivation.

Seven motivational types were found among European ayahuasca users by Schmid (2014): (a) Event type, occasional use in organized settings, workshops, or trips to South America, loosely connected to an ayahuasca network or church; (b) Therapy type, search for psychedelic therapy or alternative cure of physical issues; (c) Seeker type, search for a personal philosophy, identity, or affirmation in different philosophies; (d) Healer type, often on mission to provide ayahuasca to others, usually after personal healing experience or revelations about their future as a "healer;" (e) Spiritual type, on a search for transpersonal or spiritual experiences; (f) Substance user type, consumes ayahuasca similar to other psychoactive drugs, recreational motives and curiosity; (g) Alternative type, ecological orientation, often engaged in environmental or human rights activities for the rainforest or indigenous people, may have non-conformist personality. Holman (2010) suggests a 3-category typology for western ayahuasca drinkers directly in South America: (a) purposeful and often exclusive motivation for shamanic healing and ayahuasca; (b) motivation for different touristic activities of which the experimental ayahuasca intake is only one; (c) randomly found opportunity of ayahuasca consumption on streets - not part of any organized ayahuasca seminar. Kristensen (1998) found four general motivational categories: (a) self-exploration and spiritual growth, (b) curiosity, (c) physical and emotional healing, and (d) desire for a vacation to an exotic location.

Psychedelic tourism and the international spreading of ayahuasca strongly benefit from the Internet. Social networks play a significant role in the reinvention of ayahuasca culture and it is spreading into new contexts (Conrad, 2018). According to Facebook, the biggest closed Facebook group named "Ayahuasca" had 74,100 members (December 15, 2017) and 10 months later 81.311 members (October 2, 2018). Motives and characteristics of ayahuasca-interested individuals need more attention in order to further investigate which role they play for the experience and integration process, how they are linked together, and how they may influence ayahuasca practitioners and their corresponding working theories.

This study aims to explore the characteristics of active members of the international Facebook community of ayahuasca by an online questionnaire. Motivational aspects and their distribution within western ayahuasca drinkers that have been previously identified as relevant by heuristic qualitative methods are examined in this study by quantitative methods.

\section{METHODS}

\section{Ethics and confidentiality}

No approval of the ethical committee was required, because no experimental or other intervention was performed on the sample in this investigation. Strict anonymity was guaranteed through the whole process by the online procedure. Participants agreed to proceed by clicking "OK" after having read an informed consent form. Confidentiality and voluntary participation were explicitly assured and would be kept throughout the research and publishing procedure.

\section{Sampling and recruitment}

The self-selected sample consisted of 40 participants from ayahuasca-specific online forums. All given responses were included in the analysis.

An online questionnaire about demographic data, preexperience, and motivation for ayahuasca was developed in three languages (English, German, and Spanish). It was placed online through a German Internet-based provider (www.umfrageonline.com). Several possible Facebook groups about ayahuasca were identified and entered. Most of those groups are closed. One has to ask for admission. The research request was posted together with an explanation and the related Internet link of the informed consent form and the questionnaire. The questionnaire was accessible for 1 month. Later, the data were downloaded and deleted from the server. Members of the following Facebook groups were asked to participate:

- European Ayahuasca Forum

- Ayahuasca

- Ayahuasca Spain/España

- Ayahuasca Yage Austria

- Ayahuasca Madre Tierra Berlin

- Ayahuasca Germany

- Ayahuasca and Sjamanisme Nederland, België

- Ayahuasca and Shamanism

- Familia Yage “Ayahuasca” Barcelona (España)

- Psychoactive Experience Group DMT, Iboga, Ayahuasca, Salvia, Mushroom, Weed

- Chaman Ayahuasca; Centro Botanico Naturalista 
- Ayahuasca - Yage Patrimonio Cultural para la Nacion y la Humanidad Colombia

- Ayahuasca (Yage) Colombia

\section{Questionnaire}

Participants were asked to provide demographical data, preexperience with ayahuasca, and other mind-altering substances. We asked about the general interest in ayahuasca: "What are your main interests in having the ayahuasca/yagé experience?" Motivational elements of modern ayahuasca drinkers that were proposed by previous studies were collected, analyzed, and merged into four general categories. Each of these motivational categories was represented by only one item that had to be rated on a 6-step rating scale (from "not true" to "absolutely true"):

- "Self-awareness and/or self-exploration"

- "Solve some physical health issues"

- "Spiritual purposes"

- "Interesting or exciting experience"

Several of the proposed items could be selected. For compliance and validation purposes, semi-structured answer fields were added.

The desire for "self-awareness and/or self-exploration" was described as personal development, understand, and integrate psychological childhood trauma, heal depression, overcome social fears, confrontation with struggles in life, find meaning and purpose in life, find identity, decrease confusion, and indecision about desires and preferences.

The motivation regarding "spiritual purposes" was described as to feel one with something greater, awake the own spirituality, overcome reductionist and atheistic education, follow a "call" from ayahuasca, be part of the spiritual development of mankind, understand everything with mind and soul, reflect the development of the soul, and "to connect with the source of understanding of the game of the matrix" (participant 36).

Only two motivations to treat physical issues were qualitatively described: fibromyalgia and Alzheimer's disease.

No one reflected qualitatively about the desire for "interesting or exciting experiences."

\section{Analysis}

The data were statistically analyzed through descriptive and inference statistical procedures: (a) test of normal curve distribution by Kolmogorov-Smirnov test and Shapiro-Wilk test, non-parametric comparison of central tendencies through the Kruskal-Wallis H-test; (b) Mann-Whitney $U$ (M\&W-U) test for each possible paired combination of motivational categories (Bonferoni-corrected); and (c) correlation coefficient from Kendall (bootstrap with 1,000 samples).

\section{RESULTS}

\section{Sample characteristics}

Gender: 22 women (55\%), 16 men (40\%), and 2 unidentified by gender.
Age: range between 22 and 67 years $(S D=10.662$, average age $=39$ years).

Chosen language: 30 English (75\%), 7 Spanish (17.5\%), and 3 German (7.5\%).

Highest education: 19 had finished university (47.5\%), 13 had finished college (32.5\%), and 6 had finished high school (15\%). Two did not respond to this question.

Profession: 12 participants worked in health care (30\%). Other professions were bus driver, student, engineer, sales manager, security guard, teacher, hairdresser, designer, gas inspector, and jobless.

Drug experiences: 28 participants (70\%) have had previous experiences with other drugs and 12 were complete novices $(30 \%)$ :

- "Magic mushrooms" (psilocybin): 19 (47.5\%)

- "Ecstasy" (3,4-methylenedioxymethamphetamine): $17(42.5 \%)$

- Cannabis: 16 (40\%)

- Lysergic acid diethylamide (LSD): 15 (37.5\%)

- Alcohol: 10 (25\%)

- Peyote cactus or San Pedro cactus (mescaline): $7(17.5 \%)$

- Cocaine: 5 (12.5\%)

- "Kambo" (skin poison secretion of the giant leaf frog "Phyllomedusa bicolor" for purging therapy): 4 (10\%)

- Antidepressants: 4 (10\%)

- DMT: 3 (7.5\%)

- Ketamine or "Crystal-Meth" (N-methylamphetamine): $2(5 \%)$

- Heroin: $1(2.5 \%)$.

Counseling or other psychospiritual therapies: half of the sample had previous experience with psychotherapy or counseling.

Ayahuasca experience: 17 had consumed ayahuasca up to 10 times before $(42.5 \%)$. Twelve had drunk the beverage between 11 and 20 times $(30 \%)$. Eleven participants had never drunk ayahuasca before $(27.5 \%)$ but planned to do so.

Ayahuasca motivation (quantitative): 35 from 38 participants $(92.1 \%)$ had planned to drink ayahuasca in the future ( 2 did not respond to the question), 3 said that they would not drink any more, 27 from $38(71.1 \%)$ had planned to drink ayahuasca within the next 3 months, and 7 from $38(18.4 \%)$ had planned to take ayahuasca within the next 7-12 months.

Setting: the settings differed between healing center, shaman's or therapist's place, retreat, private home, ceremony in nature, or urban setting.

Price: 25 from 28 who had drunk before had paid for their ayahuasca intake. The price ranged between 0 and 170 USD for a single ayahuasca ceremony and up to 1,600 USD for a course of 2 weeks and eight included ayahuasca ceremonies.

An average participant of this study is a member of a closed ayahuasca Facebook group, approximately between 22 and 67 years old and holds a rather high education degree and might be preexperienced in psychedelics such as psilocybin ("magic mushrooms"), MDMA ("ecstasy"), cannabis, and LSD. It is likely that the participant has 
experience with psychotherapy or counseling and has tried ayahuasca once or a few times before. The person would probably be willing to drink ayahuasca again within the next 6 months.

\section{Motivational distribution and correlation}

The four variables were not normally distributed (Figures 1 and 2). Because the requirements for parametric inference

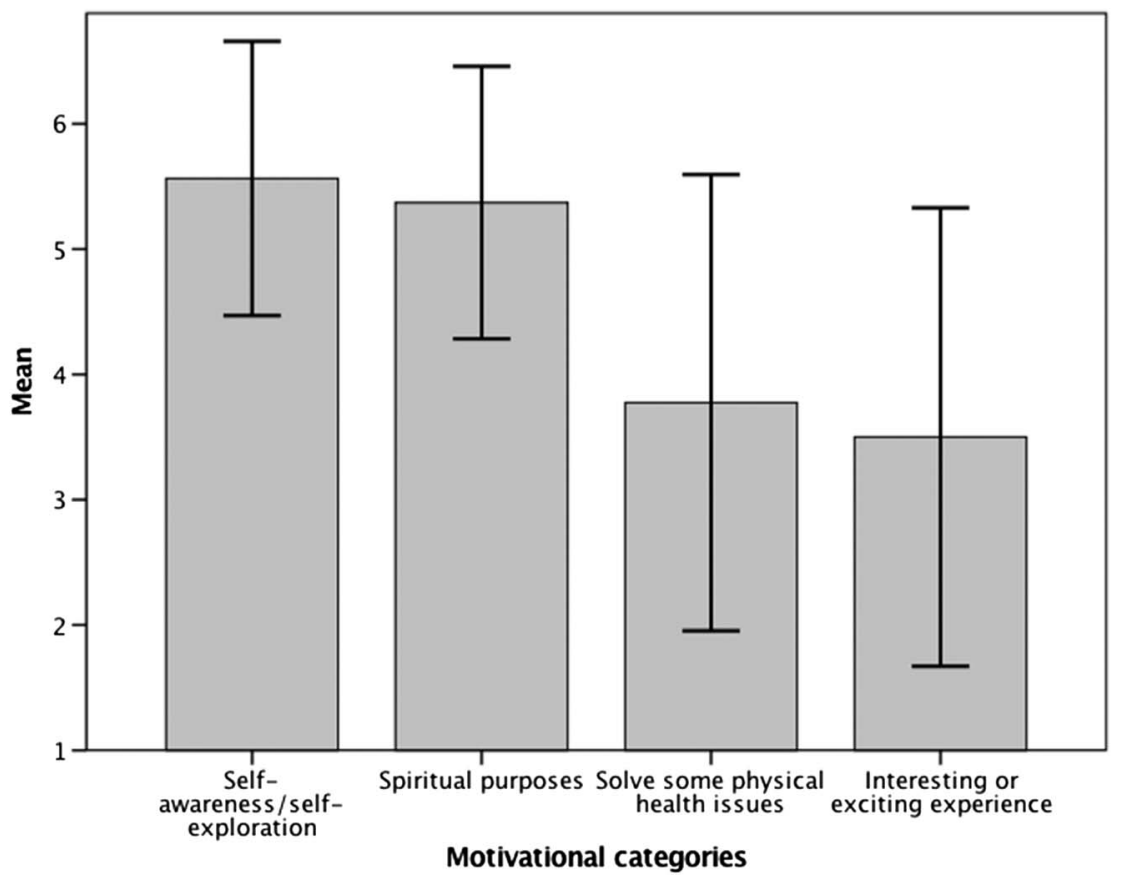

Figure 1. Motivational aspects for the ayahuasca intake in ayahuasca Facebook group members. Note: $\pm 1: S D$ of uncertainty; $Y$-axis: Likert scale from $1=$ not true to $6=$ absolutely true
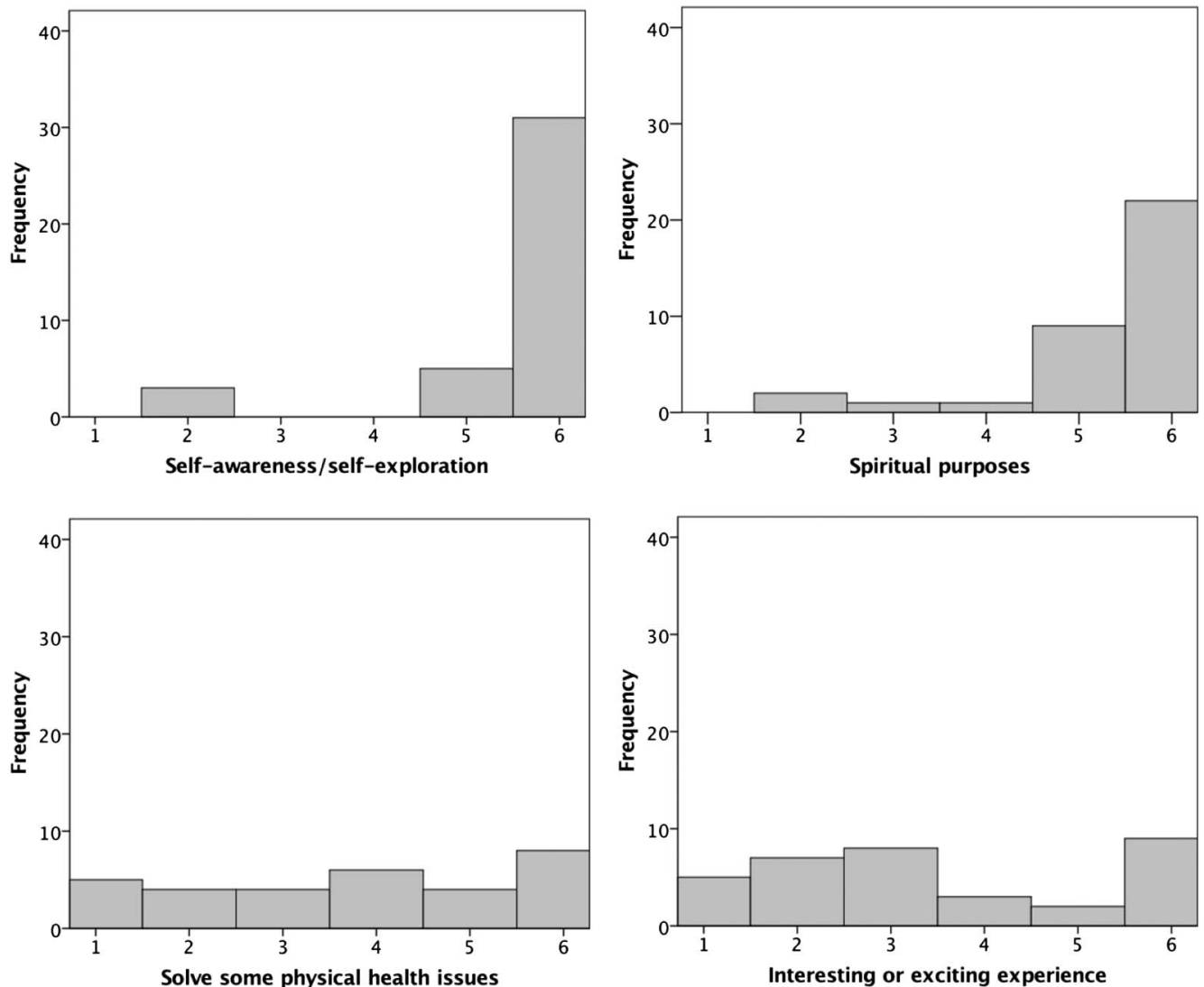

Figure 2. Histograms of different motivational aspects for the ayahuasca intake in Facebook group members. Note: $X$-axis: Likert scale from $1=$ not true to $6=$ absolutely true; $Y$-axis: frequency of participants 
statistical procedures were not met, non-parametric procedures had to be applied. The Kruskal-Wallis H-test was performed to evaluate differences among the central tendencies of the four motivations. If all assumptions of the statistical model, including the null hypothesis of no differences between these motivational elements, were true, the data could be considered unusual for this case $\left(\chi^{2}=39.071\right.$; $d f=3$; sig. $=.000$ ).

This gave evidence that either there are differences between the central tendencies or inhomogeneities of variances and distributional shapes or both.

The Kruskal-Wallis test does not provide the information, which pairs of the variables differ. In order to identify specific pairs of variables that show distribution differences, the non-parametric M\&W-U test was conducted as a twotailed post hoc test on each of the possible six combinations of the four motivational variables. Instead of an $\alpha$ level $=.05$, the Bonferoni-corrected $\alpha$ level $=.008333$ was used. The statistical null hypotheses assume that there are no differences between the distributions of pairs of variables. Significances were interpreted in combination with the mean rank differences, so that the direction of possible differences could be identified. The distribution of the motivation for "spiritual purposes" $(N=35$; mean $=5.37 ; S D=1.09)$ can be considered as high as the motivation for "self-awareness and/or self-exploration" $(N=39$; mean $=5.56 ; S D=1.095)$. These data are statistically not too unusual, assuming the hypothesis of no differences would be true $(p=.143$; $\mathrm{M} \& \mathrm{~W}-\mathrm{U}=575.5 ; Z=-1.465)$. Other assumptions of the model such as homogeneity of variances and independency of cases within the groups were fulfilled. The assumption of random sampling remained uncontrolled due to the self-selection of participants. The same applies for the combination "solving physical health issues" $(N=31$; mean $=3.77$; $S D=1.82$; mean rank $=34.44$ ) and "interesting or exciting experiences" $(N=34 ; \quad$ mean $=3.5$; $S D=1.83$; mean rank = 31.69) $(p=.552 ; \mathrm{M} \& \mathrm{~W}-\mathrm{U}=482.5$; $Z=-0.595)$.

"Solve some physical issues" scatters more in the sample than "self-awareness/self-exploration" (variance $_{\text {solve some physical issues }}=3.314$; variance $_{\text {self-awareness/ }}$ self-exploration $=1.2$ ). Participants seem more distinct in their introspective motivation than in their hope to treat physical ailments. We consider the motivation for "self-awareness/ self-exploration" (mean rank $=44.69$ ) higher than the motivation for "solving physical health issues" (mean rank= 23.94). If the null hypothesis of no differences between these distributions would be correct, the probability of having differences as seen in the data (or larger) is lower than $p=.000003$ in the case of this sample $(\mathrm{M} \& \mathrm{~W}-\mathrm{U}=246.0$; $Z=-4.673$ ). Assuming there would be no difference, these data would be unusual - either because these distributions differ from each other because of mean differences or because of inhomogeneity of variances and distributional shape or because of both reasons. The same applies for the combination "self-awareness/self-exploration" (mean rank = 47.18) and "interesting or exciting experiences" (mean rank = 25.32) $(p=.000001 ; \mathrm{M} \& \mathrm{~W}-\mathrm{U}=266 ; Z=-4.818)$.

"Solve some physical issues" also scatters more than "spiritual purposes" ( variance $_{\text {solve some physical issues }}=3.314$; variance $_{\text {spiritual purposes }}=1.182$ ). Participants seem to be more distinct in their spiritual motivation $(N=35$; mean $=$ 5.37; $S D=1.087$; mean rank $=41.54)$ rather than in their hope to treat physical ailments (mean rank $=24.42$ ). The distributions differ from each other $(\mathrm{M} \& \mathrm{~W}-\mathrm{U}=261 ; Z=$ $-3.821 ; p=.000133$ ). It cannot clearly be shown that this difference origins in the observed mean difference of 1.599 points, but it seems likely to us that the difference in these central tendencies has contributed to this finding (besides the inhomogeneity of variances).

"Interesting or exciting experiences" scatters far more in the sample than "spiritual purposes" (variance interesting or exciting experiences $=3.348$; variance $_{\text {spiritual purposes }}=1.182$ ). Participants seem more distinct in having spiritual motivations (mean rank $=44.27$ ) rather than in their interest for exciting experiences (mean rank $=25.46$ ). If the null hypothesis of no differences in the distributions would be correct (as well as all other assumptions of the model), the observed data would be very unusual $(\mathrm{M} \& \mathrm{~W}-\mathrm{U}=270.50 ; Z=-4.104 ; p=.000041)$. It cannot clearly be shown that the difference in distributions origins in the observed mean difference of 1.8714 points, but it seems likely that the difference in these central tendencies has contributed to this finding (besides the stronger inhomogeneity of variances and differences in skewness).

Two-tailed correlation procedures between the four variables were performed in order to explore the direction and strength of possible connections. In particular, we suspected a possible connection between "self-awareness/self-exploration" and "spiritual purposes" and no correlation between other combinations. If the null-hypothesis of no correlation between "self-awareness/self-exploration" and "spiritual purposes" would be correct (as well as all other assumptions of the tested model), the data would be unusual. They show a moderate correlation (Kendall's $\tau=0.463 ; p=.004 ; 0.058<$ BC $>0.727 ; N=35)$. All other combinations did not show significant correlations (Table 1). Weak, but statistically not significant, correlations were found for the variable "solve some physical health issues" combined with "interesting or exciting experiences" (Kendall's $\tau=0.218 ; \quad p=.154 ; \quad-0.131<$ $\mathrm{BC}>0.534 ; N=28)$ as well as combined with "spiritual purposes" (Kendall's $\tau=0.178 ; p=.262 ;-0.159<\mathrm{BC}>$ $0.459 ; N=30$ ). The variable "interesting or exciting experiences" also correlated weakly, but not statistically significant, with "self-awareness/self-exploration" (Kendall's $\tau=0.107$; $p=.490 ;-0.242<\mathrm{BC}>0.386 ; N=33)$. No correlation was found between "solve some physical health issues" and "self-awareness/self-exploration" (Kendall's $\tau=0 ; \quad p=1$; $-0.346<\mathrm{BC}>0.394 ; N=31$ ).

\section{DISCUSSION AND PERSPECTIVES}

The sample characteristics show that members of ayahuasca Facebook networks participating in this study were rather well-educated adults. They tend to have had previous experience with other psychedelics or cannabis as well as experience in counseling or other psychotherapies. The interest in ayahuasca experiences among Facebook ayahuasca group members in this study seems not to be a phenomenon of a recreational youth drug culture. They tend toward 
Table 1. Correlation matrix of motivational aspects for the ayahuasca intake in ayahuasca Facebook group members

\begin{tabular}{|c|c|c|c|c|c|c|}
\hline & & & $\begin{array}{l}\text { Self-awareness/ } \\
\text { self-exploration }\end{array}$ & $\begin{array}{c}\text { Solve some physical } \\
\text { health issues }\end{array}$ & $\begin{array}{l}\text { Spiritual } \\
\text { purposes }\end{array}$ & $\begin{array}{l}\text { Interesting or exciting } \\
\text { experience }\end{array}$ \\
\hline Median & & & 6 & 4 & & 3 \\
\hline $\mathrm{BC}_{95 \%}$ & & & {$[6,6]$} & {$[3,4]$} & 6 & {$[3,3]$} \\
\hline IQR & & & 0 & 4 & 1 & 4 \\
\hline \multirow[t]{16}{*}{ Kendall's $\tau \_b$} & \multirow{4}{*}{$\begin{array}{l}\text { Self-awareness/ } \\
\text { self-exploration }\end{array}$} & Corr. & 1 & & & \\
\hline & & Sig. & - & & & \\
\hline & & $\mathrm{BC}_{95 \%}$ & & & & \\
\hline & & $N$ & 39 & & & \\
\hline & \multirow{4}{*}{$\begin{array}{c}\text { Solve some } \\
\text { physical health } \\
\text { issues }\end{array}$} & Corr. & .000 & 1 & & \\
\hline & & Sig. & 1.000 & - & & \\
\hline & & $\mathrm{BC}_{95 \%}$ & {$[-.346, .394]$} & & & \\
\hline & & $N$ & 31 & 31 & & \\
\hline & \multirow{4}{*}{$\begin{array}{l}\text { Spiritual } \\
\text { purposes }\end{array}$} & Corr. & $.463 * *$ & .178 & 1 & \\
\hline & & Sig. & .004 & .262 & - & - \\
\hline & & $\mathrm{BC}_{95 \%}$ & {$[.048, .710]$} & {$[-.159, .459]$} & & \\
\hline & & $N$ & 35 & 30 & 35 & \\
\hline & \multirow{4}{*}{$\begin{array}{l}\text { Interesting or } \\
\text { exciting } \\
\text { experience }\end{array}$} & Corr. & .107 & .218 & .140 & 1 \\
\hline & & Sig. & .490 & .154 & .362 & - \\
\hline & & $\mathrm{BC}_{95 \%}$ & {$[-.242, .386]$} & {$[-.131, .534]$} & {$[-.172, .429]$} & \\
\hline & & $N$ & 33 & 28 & 32 & 34 \\
\hline
\end{tabular}

Note. IQR: interquartile range; $\mathrm{BC}_{95 \%}$ : bootstrap 95\% confidence interval (1,000 samples), bias-corrected, and accelerated.

$* * p=.01$.

the intake within organized events, such as shamanic healing ceremonies and retreat seminars. Larger samples are needed to generalize to the population level.

It can be assumed that, within the motivational structure of the sample, there is a stronger group of motivational aspects and a weaker group of motivational aspects. The stronger one contains spiritual interests and the selfexploring motivation. The weaker group contains the intention to solve health issues and to seek interesting sensations.

The two categories "self-awareness/self-exploration" and "spiritual purposes" appeared to be the most relevant in terms of the general motivation for ayahuasca within the sample. "Self-awareness/self-exploration" also comprises the psychotherapeutic motivation of healing depressive symptoms, social anxiety symptoms, and childhood trauma. On one hand, the motivations subsumed under this item seem to be past-oriented, in the sense of biographical understanding, integration, and coping with past experiences, and on the other hand, future-oriented, in the sense of hoping for guidance, recognition of own preferences in life, and meaningful shaping of the future. Another categorization would be possible: psychotherapeutic motivation, personal meaning, adjustment, and betterment. A comprehensive qualitative content analysis of the expectations of ayahuasca clients is needed.

"Self-awareness/self-exploration" and "spiritual purposes" correlated positively with each other in the sample. It is known from European neo-shamanic scenes that previously narrowly defined terms get a changed and sometimes overgeneralized meaning. In neo-shamanic circles, this can be the case with the concept of healing (Morris, 2006). Within those peer groups, the term might overlap with concepts, such as "cleansing from civilization influences" or "personal spiritual development and wisdom." In our sample, there could also be a blurring between "spiritual purposes" and "self-awareness/self-exploration." For this study, the qualitative analysis of the subjective meaning of these predetermined motivational items points toward such a partial overlap, when the hope for a "life changing experience" was expressed that would heal depression (participant 5) as well as the desire to "reflect the development of the soul" (36) or when ayahuasca was seen as "another piece ... of the spiritual journey... in the category of how certain plants can heal us" (26).

Since the variables "spiritual purposes" and "selfawareness/self-exploration" have similar distributions and are correlated, it would be interesting to further examine similarities and differences in the subjective meaning of them and how they may be connected.

In interviews with curanderos (Spanish: healer) from the upper Amazonas, who work with foreign clients, Beyer (2009, pp. 347-348) reported that they believe that, unlike local clients, foreign ayahuasca drinkers would rather have spiritual and esoterical interests and would rather be confused, tormented, and burdened with mental problems, often related to their childhood. The higher motivation for spiritual purposes and self-awareness/self-exploration in this sample and their correlation, compared with the lower motivation for solving physical issues and the lower interest in interesting or exciting experiences, does not contradict this observation.

The wider error bars of the weaker group of variables may show a greater interpersonal diversity in the motivational strength regarding the expectation of healing physical issues as well as to experience an interesting or exciting time with ayahuasca. Here, the motivations seem to be less consistent among the sample than in terms of "spiritual purposes" or "self-awareness/self-exploration."

On one hand, in terms of solving physical problems, this may also reflect the suggestion from anthropological 
literature that ayahuasca-interested foreigners may be less interested in actual physical healing than local clients (Beyer, 2009, p. 348). Yet, on the other hand, fantastic rumors about physical miracle healing through ayahuasca are repeatedly transported in the ayahuasca scene, both local and international. This may have led to a wider dispersion of expectations in the interest in physical healing.

The rather low motivational aspect of making interesting or exciting experiences surprised us. But again, the higher deviation in the sample points toward a greater interindividual variety regarding this motivational aspect.

Statistical group comparisons with larger samples between different types of ayahuasca drinkers from different settings are needed in order to complete and ensure the knowledge about the motivation of ayahuasca drinkers. Further statistical group comparisons of the motivational structure of local native and mestizo drinkers with non-native ayahuasca drinkers would be relevant for cultural anthropology to picturize the development of possible new traditions in the context of phenomena of the industrialized worlds like the individualization of spirituality and religiosity, patchwork religiosity, and how these new developments possibly influence the local motivation and ayahuasca practice in the upper Amazonas region. Further studies could better detail what ayahuasca drinkers exactly mean when they report spiritual reasons or self-exploration and how previous motivation and expectation is related to their actual trip experiences. Another promising approach would be the analysis of how possibly the motivation and expectation change from beginners toward long-term drinkers.

\section{LIMITATIONS}

The generalizability of these findings toward the whole population of non-native ayahuasca drinkers is statistically uncertain due to the active self-selection of participants within this non-experimental design without randomization and a rather small sample seize. The generalization toward the whole population of ayahuasca westerns should therefore be avoided for the present. The representativeness of the sample for the targeted population of western ayahuasca users is limited to active members of the international ayahuasca Facebook scene, who are willing to take part in such a study. It is unknown if the self-selected sample which is small compared to the great number of members of the selected of Facebook groups - overrepresents a particular part of the ayahuasca community, who might have a particular intrinsic motivation for participating in such a study that may have affected the results. More statistical data from different sources and from different types of ayahuasca drinkers are needed for a general picture of the motivational structure of ayahuasca tourism. Since the qualitative description of the given motivational categories was not mandatory, a complete picture of their subjective meaning could not be given. Especially the variable "spiritual purposes" might be overlapping with "self-awareness/ self-exploration." This may have partly contributed to the correlation of the two. A better validation of the four motivational categories beforehand would be important. Validity and reliability are unknown for the used four items. Correlation hypotheses were developed, after the data had been collected and analyzed and were not part of the original conceptual design.

Acknowledgements: TJW is a primary writer and researcher. $\mathrm{TP}$ is an editor of this manuscript. There is no financial support received for this article.

Conflict of interest: The authors declare no conflict of interest.

\section{REFERENCES}

Barbosa, P., Mizumoto, S., Bogenschutz, M., \& Strassmann, R. (2012). Health status of ayahuasca users. Drug Testing and Analysis, 4(7-8), 601-609. doi:10.1002/dta.1383

Beyer, S. V. (2009). Singing to the plants: A guide to mestizo shamanism in the Upper Amazon. Albuquerque, NM: University of New Mexico Press.

Burroughs, W., \& Ginsberg, A. (1963). The yage letters. San Francisco, CA: City Lights Publishers.

Conrad, M. (2018). The global expansion of ayahuasca through the Internet. In B. L. Labate \& C. Cavnar (Eds.), The expanding world of ayahuasca - Appropriation, integration and legislation (p. 97). New York, NY: Routledge.

De Rios, M. D. (1972). Visionary vine: Hallucinogenic healing in the Peruvian Amazon. San Francisco, CA: Chandler Publishing.

De Rios, M. D. (1994). Drug tourism in the Amazon. Antropology of Consciousness, 5(1), 16-19. doi:10.1525/ac.1994.5.1.16

Fotiou, E. (2010). From medicine men to day trippers: Shamanic tourism in Iquitos, Peru (Doctoral dissertation). Department of Anthropology, University of Wisconsin-Madison, Madison, WI, p. 126.

Fotiou, E. (2014). On the uneasiness of tourism - Considerations on shamanic tourism in Western Amazonia. In B. C. Labate \& C. Cavnar (Eds.), Ayahuasca shamanism in the Amazonas and beyond (p. 162). Oxford, UK: Oxford University Press.

Grunwell, J. N. (1998). Ayahuasca tourism in South America. MAPS Bulletin, 8(3), 59-62. Retrieved October 3, 2017, from http://www.maps.org/news-letters/v08n3/08359gru.html

Holman, Ch. L. (2010). Spirituality for sale. An analysis of ayahuasca tourism (Doctoral dissertation). Arizona State University, Tempe, AZ, pp. 8-9, 187-210.

Holman, Ch. L. (2011). Surfing for a shaman. Analyzing an ayahuasca website. Annals of Tourism Research, 38(1), 90-109. doi:10.1016/j.annals.2010.05.005

Homan, J. (2017). Disentangling the ayahuasca boom - Local impacts in Western Peruvian Amazonia. In B. C. Labate, C. Cavnar, \& A. K. Gearin (Eds.), The world ayahuasca diasporaReinvention and controversies (p. 167). New York, NY: Routledge.

Incayawar, M. (2007). Indigenous peoples in South America Inequalities in mental health care. In K. Blui \& D. Bhugra (Eds.), Culture and mental health - A comprehensive textbook (pp. 185-190). London, UK: Holder Arnold. 
Kristensen, K. (1998). The ayahuasca phenomenon: Jungle Pilgrims: North Americans participating in Amazon ayahuasca ceremonies. Retrieved June, 2018, from http://www.maps.org/ articles/5408-the-ayahuasca-phenomenon

Labate, B. C., Cavnar, C., \& Gearin, A. K. (2017). The word ayahuasca diaspora - Reinventions and controversies (p. 13). New York, NY: Routledge.

Luna, L. E. (2004). Ayahuasca ritual use. In E. J. Neumann Fridman \& M. N. Walters (Eds.), Shamanism: An encyclopedia of world beliefs, practices and cultures (pp. 378-382). Santa Barbara, CA/Denver, CO/Oxford, UK: ABC-CLIO.

Luna, L. E. (2005). Narrativas da alteridade: Aayahuasca e o motivo de transformaçãoem animal [Narratives of otherness: Ayahuasca and the motive of animal transformation]. In B. C. Labate \& S. L. Goulart (Eds.), O uso ritual das plantas de poder [The ritual use of power plants] (pp. 333-354). Campinas, Brazil: Mercado de Letras.

McKenna, D. J., Towers, G. H., \& Abbott, F. S. (1984). Monoamine-oxidase inhibitors in South-American hallucinogenic plants - Tryptamine and beta-carboline constituents of ayahuasca. Journal of Ethnopharmacology, 10(2), 195-223. doi:10.1016/0378-8741(84)90003-5

Miller-Weisberger, J. S. (2000). A Huaorani myth of the first Miiyabu. In L. E. Luna \& S. F. White (Eds.), Ayahuasca reader: Encounters with the Amazon's sacred vine. Santa Fe, NM: Synergetic Press.

Morris, B. (2006). Religion and anthroplogy, a critical introduction (p. 36). Cambridge, UK: Cambridge University Press.
Ott, J. (1994). Ayahuasca analogues: Pangcean entheogens (1st ed., pp. 81-83). Kennewick, WA: Natural Products.

Ott, J. (1996). Pharmacotheon, entheogenic drugs, their plant sources and history (pp. 212-221). Kennewick, WA: Natural Products.

Santos, R. G., Landeira-Fernandez, J., Strassman, R. J., Motta, V., \& Cruz, A. P. (2007). Effects of ayahuasca on psychometric measures of anxiety, panic-like and hopelessness in Santo Daime members. Journal of Ethnopharmacology, 112, 507-513. doi:10.1016/j.jep.2007.04.012

Schenberg, E. E., Alexandre, J. F. M., Filev, R., Cravo, A. M., Sato, J. R., Muthukumaraswamy, S. D., Yonamine, M., Waguespack, M., Lomnicka, I., Barker, S. A., \& da Silveira, D. X. (2015). Acute biphasic effects of ayahuasca. PLoS One, 10(9), e0137202. doi:10.1371/journal.pone.0137202

Schmid, J. T. (2014). Healing with ayahuasca: Notes on therapeutic rituals and effects in European patients treating their diseases. In B. Labate \& C. Cavnar (Eds.), The therapeutic use of ayahuasca (pp. 77-93). Berlin, Germany: Springer-Verlag.

Schultes, R. E. (1983). Richard Spruce: An early ethnobotanist and explorer of the northwest Amazon and northern Andes. Journal of Ethnobiology, 3(2), 139-147. Retrieved February 1, 2018, from https://ethnobiology.org/sites/default/files/pdfs/ JoE/3-2/Schultes1983.pdf

Znamenski, A. A. (2007). The beauty of the primitive: Shamanism and the western imagination (pp. 155-157). Oxford, UK: University Press. 